\title{
Clinical Evaluation of Rotatory Gross Section Radiography (Rotatory Crossgraphy) applied to Chest Diseases
}

(Studies on Rotatography. 11th Report)

\author{
By
}

\author{
Shinji Takahashi and Tadayoshi Matsuda \\ (高 橋 信 次) \\ (松 田 忠義) \\ From the Department of Radiology, Hirosaki University \\ School of Medicine, Hirosaki
}

(Received for publication, December 9, 1952)

Since Rotatory Cross Section Radiography was invented by Vallebona, ${ }^{11}$ Gebauer, ${ }^{21}$ or Takahashi, ${ }^{31}$ it has been hoped that this method can be applied to the full field of clinical medicine. However, there have not been yet published sufficient data as expected. Studying on this theme since December 1949, we have had a pretty wide experience and have come to a conclusion about the evaluation of this method. We will detail about the theme below.

\section{Technique and Materials}

584 patients, suffering from chest disease, were taken normal roentgenograms. Then they were rotatory-crossgraphed by means of the same apparatus and the technique in the former issue of this Journal.4) And 867 roentgenograms, revealing some significant lesions in the chest, were obtained.

The ages of the patients ranged from three to seventy years.

\section{Results}

The diagnosis of the disease was established first with the normal roentgenogram, then that diagnosis was checked with the Rotatory Cross Section Radiogram.

If the latter found the additional findings and contributed a better diagnosis, this method was estimated as useful. We classified these incidences into four groups as follows.

A. Group applied to detecting lesions

126 out of 867 roentgenograms were estimated as useful in detecting lesions (Tab. I). 


\section{TABLE I}

Name of lesions detected

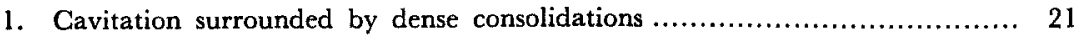

2. Cavitation hidden by shadow of the clavicula, ribs or scapulae ............. 12

3. Intrapulmonary lesions surrounded by pleural lesions ....................... 17

4. Lesions situated in the vicinity of or in mediastinum superior

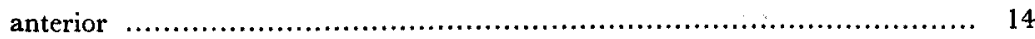

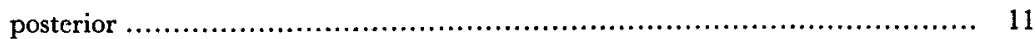

5. Intrapulmonary lesions hidden by the heart displaced or dilatated ........... 11

6. Paravertebral lesions hidden by the diaphragmatic arch..................... 5

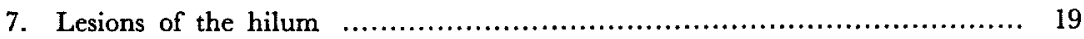

8. Lesions hidden by the mediastinum displaced by artificial pneumothorax, thoracoplasty or old pleurisy

There were three types of lesions in this group, namely, the one hidden by an extrapulmonary shadow, the intrapulmonary one surrounded by a dense shadow and the one situated at the inlet of the mediastinum. The first one might be found out easily in an oblique or a lateral view of normal roentgenography. The second one would be discovered of its true nature by means of tomography too.

Nevertheless the third one localized either in or in the vicinity of the upper mediastinum or paravertebral lesion hidden by the diaphragmatic arch might not be found out by the tomographic procedure due to the occurrence of nucleus shadows. This area was made clear only by the Rotatory Cross Section Radiography.

B. Group applied to differential diagnosis

214 out of 867 cross section roentgenograms were useful to establish the proper diagnosis. (Tab. II).

\section{TAB LE II}

\section{Name of lesions differentiated}

1. Cavitation imaged in a ring figure and suspected as superimposition of shadows

2. Lesions superimposed at the area of the hilum and differentiated as : lymphadenitis.

perihilar infiltration and intrapulmonary lesions.......................... 12

3. Opacity with sharp border, differentiated as:

encapsulated pleurisy

athelectasis

pneumonia

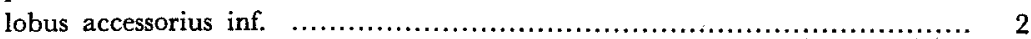

4. Cases misdiagnosed as the intrapulmonary lesions and later proved to be:

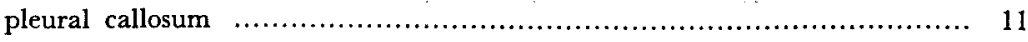

encysted pleurisy ........................................................ 10

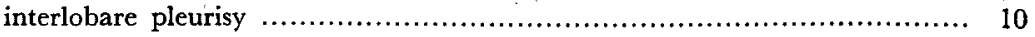




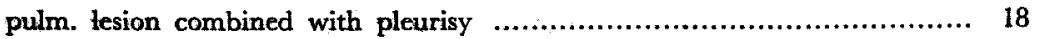

pulm. infiltration ..................................................................... 8

5. Enlargement of the upper mediastinum found due to:

anterior mediastinal pleurisy .................................................... 24

paratracheal lymphadenitis ........................................................ 12

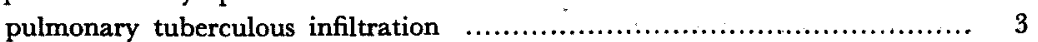

thymus hypertrophy ............................................................. 4

6. Miscellaneous,

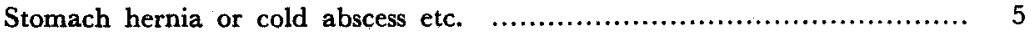

This result would not have been obtained by an ordinary roentgenography only, because the differential diagnosis in this group has been difficult to be established until the location of lesions was made clear. For this purpose either the Rotatory Cross Section Radiography or the normal roentgenography in oblique or lateral view was suitable. The latter, however, would fail to image lesions of the mediastinum clearly.

There were contained other cases in this group in which ring shadow imaged on the pulmonary field of a normal roentgenogram was suspected as a mere superimposition of line shadows. This was differentiated too as a image of cavitation when that was imaged as a ring shadow on both roentgenograms which were taken by normal roentgenography and by Rotatory Cross Section Radiography.

C. Group applied to the determination of the extent and position of lesions (Table III)

505 out of 867 roentgenograms were useful to determine the site, extent or position of lesions.

\section{T A B L E III}

Name of lesions made clear of their location etc, Cases

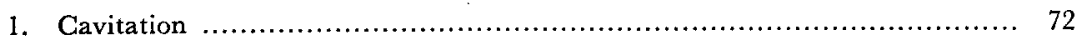

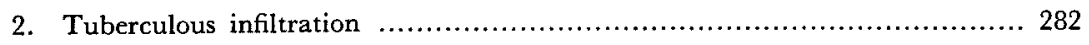

The one accompanied by the atrophy of the thorax $\ldots \ldots \ldots \ldots \ldots \ldots \ldots \ldots \ldots, 21$

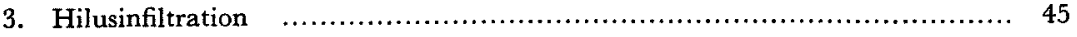

4. Pleurisy,

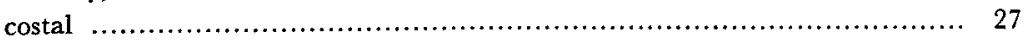

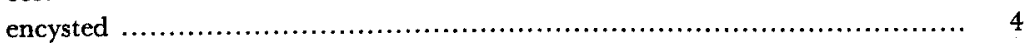

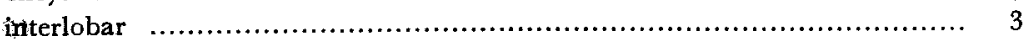

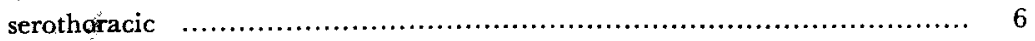

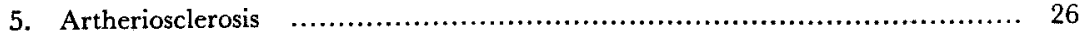

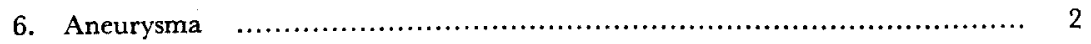

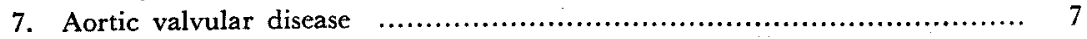

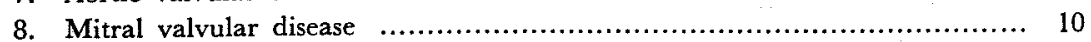

Concerning the diagnosis these lesions had already been diagnosed properly with the normal roentgenogram. Either the state of the thoracic 
atrophy or the deformity of the heart caused by the valvular diseases is manifested more cleary on occasion by this roentgenography. Moreover three dimensional knowledge about the lesions has never been learned by a normal roentgenographic procedure. From this point of view this method was estimated as useful.

D. Group applied to the pretherapeutic procedure

153 out of 867 roentgenograms served us not only in diagnostic use but also in therapeutic one (Tab. IV).

\section{TAB LE IV}

Name of treatment of lesions, surgical or radiological

1. Collapstherapy in determining the state of a collapsed lung after:

thoracoplasty...

extrapleural prombaging or pneumosewing...................................... 8

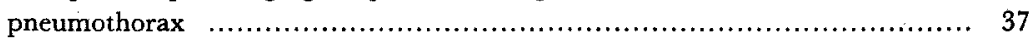

2. Determination of the location of foreign body ............................. 6

3. Punction of the exudative pleurisy or the cavitation $\ldots \ldots \ldots \ldots \ldots \ldots \ldots \ldots \ldots . . .16$

4. x-ray treatment of lung tumors adjusted with isodose chart.

pulmonary or broncheal tumor.......................................... 25

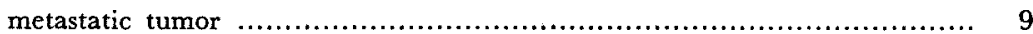

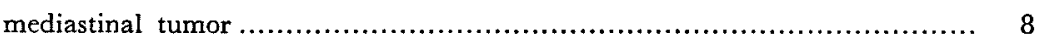

The collapsed lung adhered to the mediastinum in pneumothorax, or the one displaced to the retrovertebrale sinus in thoracoplasty was found out easily by this method.

The location of foreign bodies was most easily determined.

In planning the irradiation to the pulmonary tumor we applied the isodose chart to the cross section of the chest. Without the Cross Section Radiogram the reasonable irradiation would not be performed.

\section{Discussion}

As for profitable features of the Rotatory Cross Section Radiography, Takahashi, ${ }^{45)}$ like Vallebona ${ }^{6)}$ and Gebauer, ${ }^{71}$ has pointed out from his own experience that this method was superior to the customary roentgenography because the Cross Section Radiography enabled us to obtain the image of cross section of the body, and it was supperior also to tomography because that method made clear the state of the mediastinum. This is now proved here clearly. Moreover the merits of this method, when applied to chest diseases, were enumerated one by one. Concerning its ability in detecting lesions except the one in the vicinity of the mediastinum, the Rotatory Cross Section Radiography may be a little inferior to tomography due to its fuzzy images, but it can show more directly the location 
and the shape of lesions in the cross section.

In differentiating the diagnosis of illness, this method serves us just as effectively as the oblique view of the normal roentgenography.

It was learned actually that the real diagnosis is to be established upon the acknowledgment of the location or the shape of lesions in the chest.

Moreover this methode has advantage that it can be applied to the area of pretherapeutic procedure.

The roentgenograms taken with our technique (small inclination of central x-ray of $15^{\circ}$ to the film) reveals the state of the upper pulmonary field free from the obstructive shadow (or line shadow ${ }^{8}$ ) of ribs, which is often seen in the image taken with other techniques than ours.

Thus at least as regards the lesions located in the apex or in the vicinity of the mediastinum our technique can be said to be more effective than other techniques. A pretty big number of 584 of the patients upon which our experiments were based seems to strengthen our conclusion.

\section{References}

1) Vallebona, A., Radiol. Sperim., 1948, 2, 95.

2) Gebauer, A., Fortschr. Roentgenstr. 1949, 71, 669.

3) Takahashi, S., Tohoku J. Exp. Med., 1950, 52, 138.

4) Takahashi, S., ibid., 1951, 54, 59.

5) Takahashi, S., Hirosaki Med. J. (Japanese), 1951,|2, 1.

6) Vallebona, A., L' Informatore Medico, 1948, $2,1$.

7) Gebauer, A., Fortschr. Roentgenstr., 1951, 75, 9.

8) Takahashi, S., Tohoku J. Exp. Med., 1953, 58, 1. 